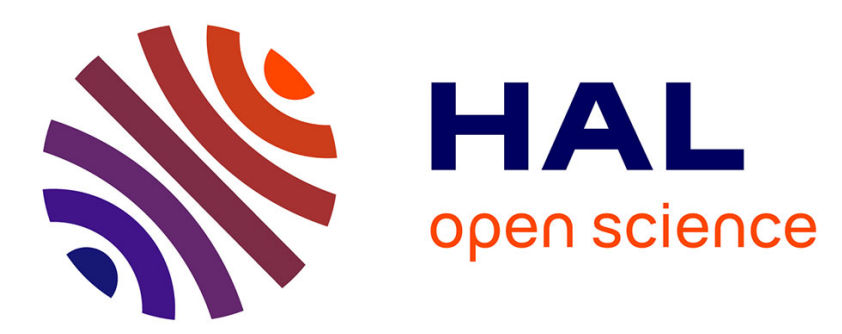

\title{
Réponse de la photosynthèse de deux variétés de blé à un déficit hydrique foliaire
}

Claire Aboussouan-Seropian, Claude Planchon

\section{To cite this version:}

Claire Aboussouan-Seropian, Claude Planchon. Réponse de la photosynthèse de deux variétés de blé à un déficit hydrique foliaire. Agronomie, 1985, 5 (7), pp.639-644. hal-00884794

\section{HAL Id: hal-00884794 \\ https://hal.science/hal-00884794}

Submitted on 1 Jan 1985

HAL is a multi-disciplinary open access archive for the deposit and dissemination of scientific research documents, whether they are published or not. The documents may come from teaching and research institutions in France or abroad, or from public or private research centers.
L'archive ouverte pluridisciplinaire HAL, est destinée au dépôt et à la diffusion de documents scientifiques de niveau recherche, publiés ou non, émanant des établissements d'enseignement et de recherche français ou étrangers, des laboratoires publics ou privés. 


\title{
Réponse de la photosynthèse de deux variétés de blé à un déficit hydrique foliaire
}

\author{
Claire ABOUSSOUAN-SEROPIAN \& Claude PLANCHON \\ Ecole Nationale Supérieure Agronomique, Photosynthèse et Amélioration des Plantes, 145, avenue de Muret, \\ F 31076 Toulouse Cedex
}

RÉSUMÉ

\begin{abstract}
La comparaison de 2 variétés de blé, « Bidi 17 " (Triticum durum) et «Capitole » ( $T$. aestivum), différant par leur capacité à tolérer les faibles potentiels hydriques foliaires, permet de montrer que les phénomènes stomatiques et non stomatiques concourent parallèlement à la dépression de la photosynthèse au cours d'un stress hydrique. Les phénomènes photorespiratoires apparaissent moins affectés par le déficit hydrique que la photosynthèse, le point de compensation $(\Gamma)$ pour le $\mathrm{CO}_{2}$ augmentant avec l'abaissement du potentiel hydrique foliaire en fonction de la réponse de la photosynthèse nette du génotype au déficit hydrique. Le rendement lumineux maximal $(\alpha)$ diminue à partir des potentiels hydriques foliaires modérés $(-10$ bars) chez la variété sensible à la sécheresse. Tous les paramètres étudiés, liés aux processus d'échanges gazeux, sont favorables à la variété résistante, en accord avec les données agronomiques connues.
\end{abstract}

Mots clés additionnels : Conductance stomatique, résistance stomatique, potentiel hydrique foliaire, photorespiration, rendement lumineux maximal.

\section{Photosynthetic response of two wheat cultivars to water stress.}

Comparison of two wheat cultivars, 'Bidi 17' (Triticum durum) and 'Capitole' (Triticum aestivum) with different tolerance to low leaf water potentials showed that both stomatal and non-stomatal processes led to a depression in photosynthesis during a water stress event. Photorespiratory processes seemed to be less affected by water stress than photosynthesis ; the $\mathrm{CO}_{2}$ compensation concentration $(\Gamma)$ increased when the leaf water potential decreased, according to the photosynthesis response of the cultivar to water stress. The maximum efficiency of light energy conversion $(\alpha)$ decreased with leaf water potential, as soon as moderate values were reached ( -10 bars) in the drought-sensitive cultivar. All investigated parameters related to gaseous exchange processes showed the resistant variety to be more efficient, in agreement with known agronomical data.

Additional key words : Stomatal conductance, stomatal resistance, leaf water potential, photorespiration, maximum efficiency of light energy conversion.

\section{INTRODUCTION}

Pour les plantes cultivées, la résistance à la sécheresse désigne l'aptitude à produire malgré des disponibilités en eau limitées. Les voies d'adaptation au déficit hydrique sont diverses (BEGG \& TURNER, 1976; TURNER et al., 1978). Presque tous les aspects de la croissance sont affectés par le déficit en eau (HSIAO, 1973), en particulier la photosynthèse, facteur primaire de la production totale. Parallèlement, la vitesse d'exportation des photosynthétats dont dépend le rendement en grains est rapidement diminué chez le blé, au cours du déficit hydrique (WATSON \& WARDLAW, 1981). Il existe, de plus, chez le blé tendre, une bonne relation entre l'activité chlorophyllienne de la dernière feuille, après la floraison, et le rendement en grains (PLANCHON, 1976).

La réduction de la photosynthèse, comme celle de la transpiration, liée à la diminution du potentiel hydrique foliaire, est attribuée initialement à une fermeture des stomates, avec pour conséquence une augmentation de la résistance à la diffusion du $\mathrm{CO}_{2}$. Cependant la pénétration du $\mathrm{CO}_{2}$ peut être limitée par un accroissement de la résistance intracellulaire, sans que les causes soient actuellement parfaitement élucidées (LAWLOR, 1976). De même les réactions dans les chloroplastes, spécialement les phénomènes lumineux (activité du photosystème II, transport des électrons) peuvent être affectées chez certaines espèces (Helianthus annuus L., Xanthium strumarium L.) même 
pour des stress hydriques modérés (BOYER, 1971; SHARKEY \& BADGER, 1983). Les effets non stomatiques peuvent être importants au cours de déficits hydriques rapides, en relation avec la teneur en $\mathrm{CO}_{2}$ et certaines modalités de stress chez Sinapis alba L. (CORNIC et al., 1983). Chez le maïs, les membranes chloroplastiques sont affectées de façon irréversible par un déficit hydrique foliaire (HAVAUX \& LANNOYE, 1983).

Notre objectif est une approche des modalités de la résistance à la sécheresse mettant en jeu les échanges gazeux au niveau foliaire, à partir de protocoles de mesures assez simples permettant une application à la sélection du blé. L'étude conduite sur 2 variétés de blé connues pour leur comportement agronomique différent vis-à-vis de la sécheresse, devrait permettre de mieux préciser certains aspects de l'action d'un déficit hydrique sur les processus de la photosynthèse.

\section{MATÉRIEL ET MÉTHODES}

\section{A. Matériel végétal et modalités d'application du stress hydrique}

Deux variétés sont étudiées : "Bidi 17 », variété de blé dur présentant une bonne résistance à la sécheresse, et "Capitole ", variété de blé tendre plus sensible. Les plantes des 2 variétés sont cultivées sous abri vitré à toit amovible avec des conditions de température et de lumière proches du plein champ, dans des pots en plastique de $18 \mathrm{~cm}$ de diamètre et de $17 \mathrm{~cm}$ de hauteur. Le substrat utilisé est un mélange sableterreau dans la proportion $2 / 1$ en masse. L'arrosage se fait régulièrement à la capacité au champ.

Les mesures sont réalisées à 2 stades après application du stress hydrique dans les conditions suivantes :

1. Stade végétatif : arrêt de l'arrosage lorsque la feuille de $6^{\mathrm{e}}$ rang commence à pointer et mesure sur les feuilles de $7^{\mathrm{e}}$ et $8^{\mathrm{e}}$ rangs, la feuille de rang supérieur étant en croissance, pendant une période d'environ $15 \mathrm{j}$.

2. Stade floraison : arrêt total de l'arrosage après un dernier apport réalisé à la floraison à la capacité au champ. Compte-tenu des conditions climatiques ambiantes et de la taille du pot, l'installation du stress est rapide $(5$ à $6 \mathrm{j})$.

L'état de dessèchement du sol est suivi par son humidité pondérale. Dans les 2 cas, des témoins sont maintenus à la capacité au champ.

\section{B. Dispositif de mesure}

La mesure du potentiel hydrique foliaire $\psi \mathrm{f}$ est faite par la méthode de la chambre à pression de SCHOLANDER et al. (1965). Les déterminations de photosynthèse nette (PN) sont réalisées à partir d'un dispositif comprenant un analyseur à infra-rouge $\mathrm{CO}_{2}$ (type Maihak Unor $4 \mathrm{~N}$ ), une chambre d'assimilation transparente et un système de conditionnement de l'air. Les caractéristiques de l'air entrant dans la chambre d'assimilation sont : teneur en $\mathrm{CO}_{2}, 320 \mathrm{vpm}$; humidité relative 35 p. 100 ; température $25^{\circ} \mathrm{C}$; énergie lumineuse reçue par la feuille, $140 \mathrm{~W} \cdot \mathrm{m}^{-2}$ (PAR). Pour la mesure du rendement lumineux maximal $(\alpha)$, les niveaux d'éclairement (PAR) utilisés (lampe Philips HPLR $400 \mathrm{~W}$ ) sont : 8,$4 ; 14 ; 22,4 ; 28 ; 42$; $56 ; 84$ et $140 \mathrm{~W} \cdot \mathrm{m}^{-2}$.

La détermination du point de compensation pour le $\mathrm{CO}_{2}(\Gamma)$ a été obtenue par la méthode d'équilibre, l'air passant dans la chambre étant recyclé en circuit fermé grâce à une pompe, avec un éclairement de $140 \mathrm{~W} \cdot \mathrm{m}^{-2}$ (PAR). La résistance stomatique (rs) est analysée à l'aide d'un poromètre à diffusion de vapeur d'eau (Delta T - Devices MKIII) sur la partie médiane de la feuille de chaque côté de la nervure principale. La comparaison avec les résultats obtenus à partir de mesures directes de transpiration à l'aide d'un hygromètre à point de rosée (EG-G, 911 Dew All) montre une bonne concordance entre les valeurs obtenues.

\section{RÉSULTATS}

\section{A. Photosynthèse et déficit hydrique}

\section{Stade végétatif}

En bonnes conditions d'alimentation hydrique, les 2 variétés n'ont pas une photosynthèse significativement différente $\left(14,8 \pm 0,8 \mathrm{mg} \mathrm{CO} \mathrm{CO}^{-1} \mathrm{dm}^{-2}\right.$ pour « Bidi 17 » contre $14,2 \pm 0,7$ pour " Capitole »). A ce stade, au cours d'un déficit hydrique, il existe une

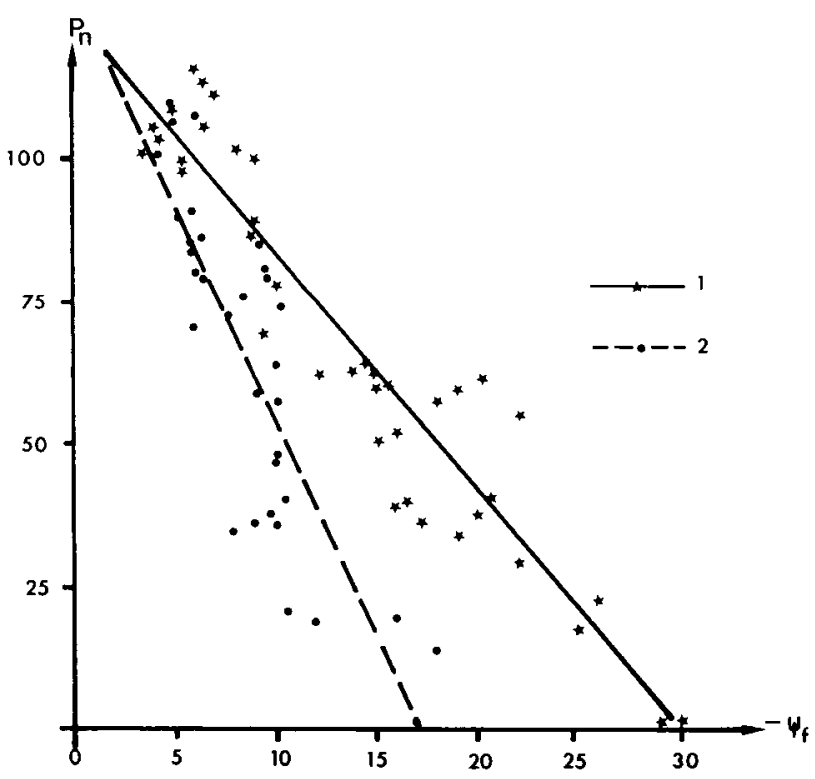

Figure 1

Relation entre la photosynthèse nette $(P N$, exprimée en \% des témoins bien arrosés) et le potentiel hydrique foliaire ( $\psi f$, bars) au stade végétatif.

(1) Bidi $17 P N=126-4,25(-\psi f) \quad r=-0,94^{x x}$

(2) Capitole $P N=131-7,62(-\psi f) \quad r=-0,85^{x x}$

Pente des droites de régression significativement différentes au seuil de $1 \%$ oo.

Relationship between net photosynthesis (PN, in \% by comparison with the check) and leaf water potential ( $\psi f$, bar).

Slopes of the regression lines of the two genotypes significantly different at $P<0.01$ 
corrélation hautement significative entre la photosynthèse nette et le potentiel hydrique foliaire. La droite de régression $P_{N}=A+B(-\psi \dot{f})$ est représentée pour les 2 génotypes (fig. 1). La photosynthèse nette s'annule pour un potentiel hydrique foliaire de - 17 bars chez "Capitole " et de - 29 bars pour « Bidi 17 ». La relation linéaire entre la photosynthèse nette et le potentiel hydrique foliaire, observée à ce stade au cours du déficit hydrique, est liée probablement à la résistance stomatique relativement élevée pour des potentiels faibles (tabl. 1).

TABLEAU 1

Résistances stomatiques des faces supérieure et inférieure $\left(s . m^{-1}\right)$ aux 2 stades de mesure, en conditions de bonne alimentation en eau. Stomatal resistance to $\mathrm{H}_{2} \mathrm{O}$ diffusion for upper and lower leaf surfaces at the 7-8 leaf and flowering stages for adequately watered plants.

\begin{tabular}{lcccr}
\hline \multicolumn{1}{c}{ Stade } & \multicolumn{2}{c}{$7-8$ feuilles } & \multicolumn{2}{c}{ Floraison } \\
\hline Face de la feuille & Inf. & Sup. & Inf. & \multicolumn{1}{c}{ Sup. } \\
\hline Bidi 17 & $330 \pm 109$ & $137 \pm 22$ & $173 \pm 54$ & $68 \pm 10$ \\
Capitole & $900 \pm 350$ & $176 \pm 75$ & $277 \pm 57$ & $118 \pm 30$ \\
\hline
\end{tabular}

Moyenne de 10 mesures \pm l'écart-type.

Values are means \pm one standard error of the mean of 10 observations.

\section{Stade floraison}

En absence de déficit hydrique, «Bidi 17 » présente une meilleure photosynthèse que «Capitole" à ce stade $\left(20,5 \pm 1,2 \mathrm{mg} \mathrm{CO} \mathrm{Ch}^{-1} \cdot \mathrm{dm}^{-2}\right.$ pour « Bidi 17 » contre $17,3 \pm 0,9$ pour « Capitole »). L'évolution du taux de photosynthèse nette en fonction du potentiel hydrique foliaire n'est pas linéaire (fig. 2). Le comportement relatif des 2 génotypes est identique à celui observé au stade végétatif mais avec une annulation de la photosynthèse pour des potentiels hydriques foliaires nettement plus faibles $(-29$ bars pour "Capitole », - 32 bars pour « Bidi 17 ») (tabl. 2). La plante conserve une activité assimilatrice pour des déficits hydriques plus sévères à ce stade.

\section{B. Action du déficit hydrique sur les différents para- mètres de la photosynthèse}

\section{Résistance, conductance stomatique et potentiel hydrique foliaire}

Chez le blé, la résistance à la diffusion de l'eau et du $\mathrm{CO}_{2}$ de la face abaxiale est plus variable et nettement supérieure à celle de la face adaxiale (MORGAN, 1977). Ce point a été vérifié pour les 2 variétés étudiées et pour les 2 stades (tabl. 1). De plus, la fermeture des stomates pour la face inférieure survient pour des potentiels hydriques foliaires plus élevés (tabl. 2). Ainsi, comme l'ont fait un certain nombre d'auteurs (FRANK et al., 1973 ; SIMMELSGAARD, 1976 ; QUARRIE \& JONES, 1979), nous utiliserons la résistance foliaire de la face adaxiale comme critère de comportement de la résistance totale.

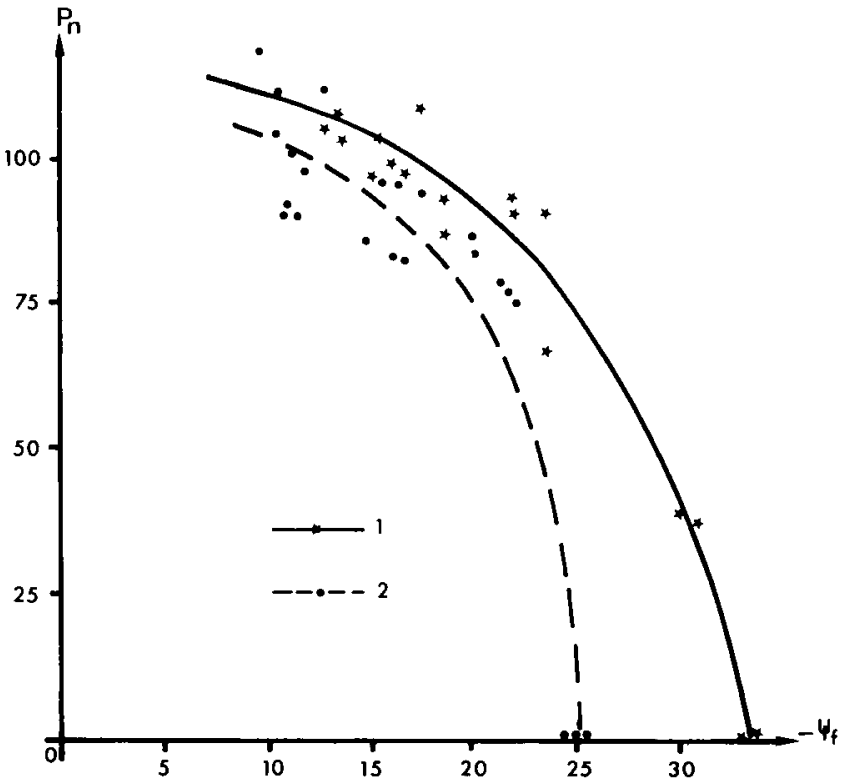

Figure 2

Relation entre la photosynthèse nette ( $P N$, exprimée en \% des témoins bien arrosés) et le potentiel hydrique foliaire ( $\psi f$, bars) au stade floraison.

$\begin{array}{ll}\text { (I) Bidi } 17 & \text { (2) Capitole. }\end{array}$

Relationship between net photosynthesis $(P N$, in \% by comparison with the check) and leaf water potentiel ( $\psi f$, bar, at the flowering stage).

\section{TABLEAU 2}

Potentiel hydrique foliaire critique (bars) de fermeture stomatique des faces supérieure et inférieure des 2 variétés aux 2 stades de mesure.

Leaf water potentiel $(\psi f$, bar) at the critical phase of stomatal closure of the upper and lower leaf surfaces (stages : 7-8 leaf and flowering).

\begin{tabular}{|c|c|c|c|c|}
\hline \multirow{2}{*}{$\frac{\text { Stade }}{\text { Face de la feuille }}$} & \multicolumn{2}{|c|}{$7-8$ feuilles } & \multicolumn{2}{|c|}{ Floraison } \\
\hline & Inf. & Sup. & Inf. & Sup. \\
\hline Bidi 17 & $\begin{array}{r}-12 \\
-\quad 9\end{array}$ & $\begin{array}{l}-29 \\
-16\end{array}$ & $\begin{array}{l}-25 \\
-20\end{array}$ & $\begin{array}{r}-32 \\
-35\end{array}$ \\
\hline & & & & \\
\hline
\end{tabular}

Valeurs obtenues à partir des courbes conductance stomatiquepotentiel hydrique foliaire, représentées uniquement à titre d'exemple pour la face supérieure au stade floraison (fig. 4).

Values obtained from the stomatal conductance-leaf water potentiel relationships (fig. 4).

Les relations conductance ou résistance stomatique potentiel hydrique foliaire sont analysées pour les 2 variétés et permettent de déterminer le potentiel critique de fermeture des stomates.

\section{a) Stade végétatif}

Nous avons linéarisé les courbes (résistance stomatique de la face supérieure - potentiel hydrique foliaire) par transformation logarithmique. Les coefficients sont significatifs au seuil de 1 p. 100 et les pentes des droites des variétés significativement différentes au seuil de 1 p. 100 (fig. 3).

La valeur du potentiel hydrique foliaire critique de fermeture stomatique diffère également entre les 2 variétés (tabl. 2). 


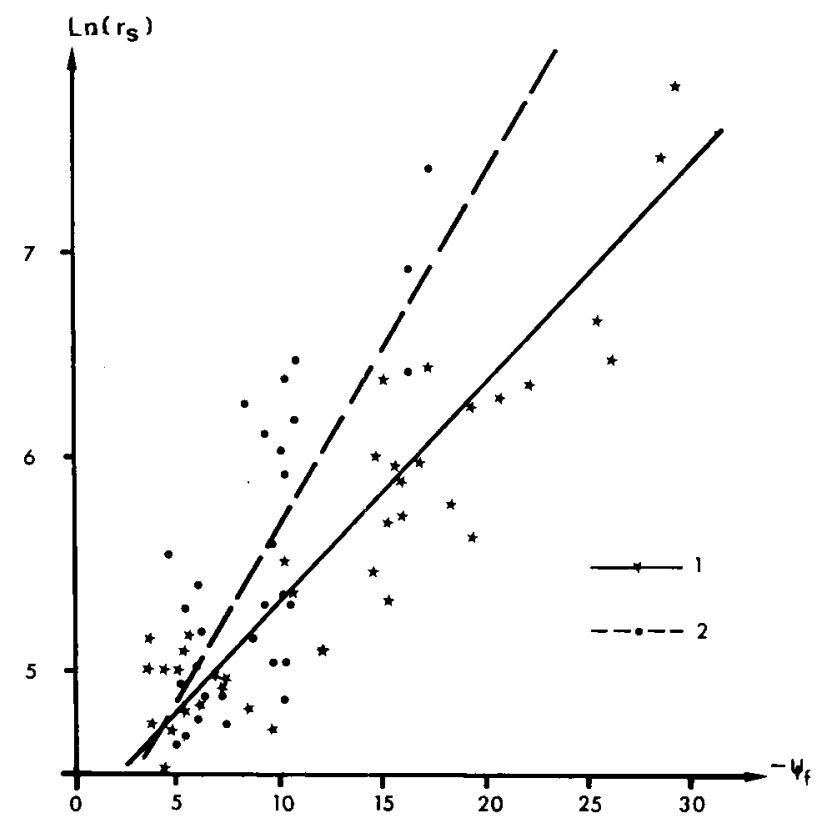

Figure 3

Relation entre la résistance stomatique de la face supérieure (exprimée par I n, $r_{\text {su }}$, s. $\mathrm{m}^{-l}$ ) et le potentiel hydrique foliaire ( $\psi f$, bars) au stade 7-8 feuilles.

(1) Bidi 17 In. $r_{s u}=4,23+0,107(-\psi f) \quad r=0,84^{x x}$

(2) Capitole 1 n. $r_{s u}=4,05+0,167(-\psi f f) \quad r=0,77^{x x}$

Pentes des droites de régression significativement différentes au seuil de $1 \%$ oo.

Relationship between stomatal resistance to $\mathrm{H}_{2} \mathrm{O}$ diffusion for upper leaf surface ( 1 n. $\left.r_{s u} ; s . m^{-1}\right)$ and leaf potential $(-\psi f)$ at 7 8-leaf stage. Two cultivars significantly different at $P<0.01$.

\section{b) Stade floraison}

La fermeture stomatique survient pour des potentiels hydriques foliaires plus faibles qu'au stade végétatif, mais les 2 variétés se classent dans le même ordre (fig. 4 et tabl. 2), Les stomates de la dernière feuille après floraison apparaissent moins sensibles au déficit hydrique que ceux des feuilles 7-8, résultat en accord avec ceux de FRANK et al. (1973) (tabl. 2). La conductance stomatique diminue assez régulièrement au cours de déficits hydriques foliaires modérés, en particulier pour «Bidi 17 » (fig. 4) et plus rapidement que la photosynthèse nette (fig. 2) pour laquelle les autres résistances au transfert du $\mathrm{CO}_{2}$ conservent un rôle important.

\section{Point de compensation pour le $\mathrm{CO}_{2}$}

Les mesures ont été effectuées au seul stade végétatif. Les 2 variétés ne présentent pas de différences significatives en conditions de bonne alimentation hydrique $(46,5 \pm 5 \mathrm{vpm}$ pour «Bidi $17 » ; 50 \pm 5$ pour «Capitole »). Par contre, lors d'un déficit hydrique, elles ont un comportement nettement différent (fig. 5), « Bidi 17 », variété résistante à la sécheresse, présentant une moindre augmentation du point de compensation pour le $\mathrm{CO}_{2}$ avec l'abaissement du potentiel hydrique foliaire. Il apparaît un effet non stomatique du déficit hydrique sur la photosynthèse nette du blé lié aux processus de carboxylation et de décarboxylation. La photorespiration diminue probablement moins vite que la photosynthèse réelle au cours du déficit hydrique, ceci étant plus net pour la

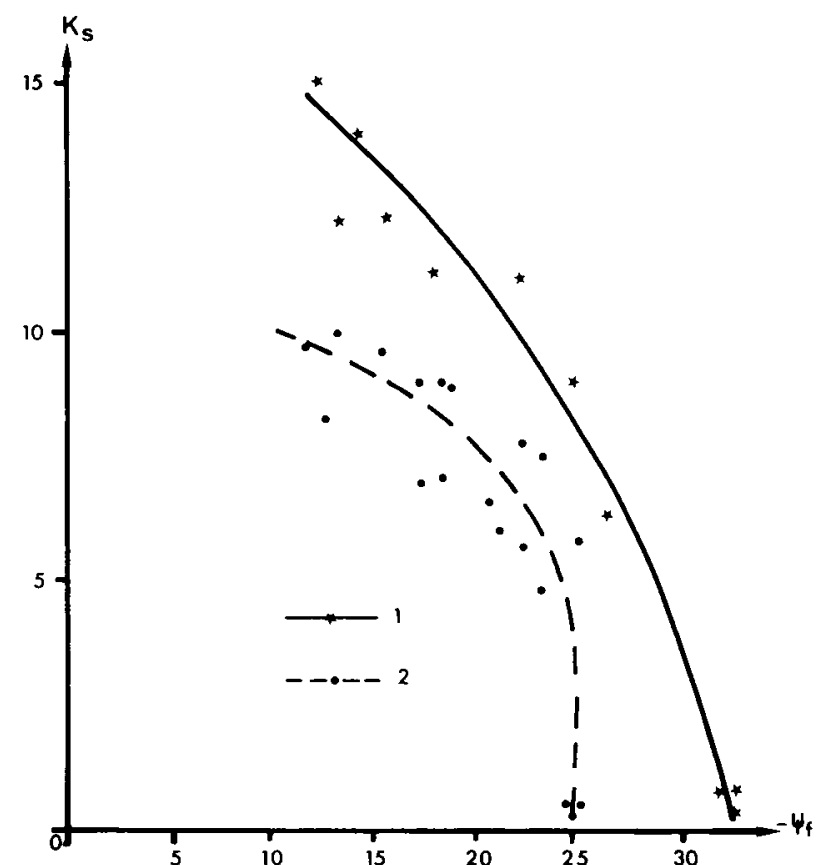

Figure 4

Relation entre la conductance stomatique de la face supérieure de la feuille $\left(K_{s} 10^{-3} \mathrm{~m} . \mathrm{s}^{-1}\right)$ et le potentiel hydrique foliaire $(\psi f$, bars $)$ au stade floraison.
(1) Bidi 17
(2) Capitole.

Relationship between stomatal conductance to $\mathrm{H}_{2} \mathrm{O}$ diffusion for upper leaf surface $\left(K_{s} m . s^{-1}\right)$ and leaf water potential ( $\psi f$, bar) at the flowering stage.

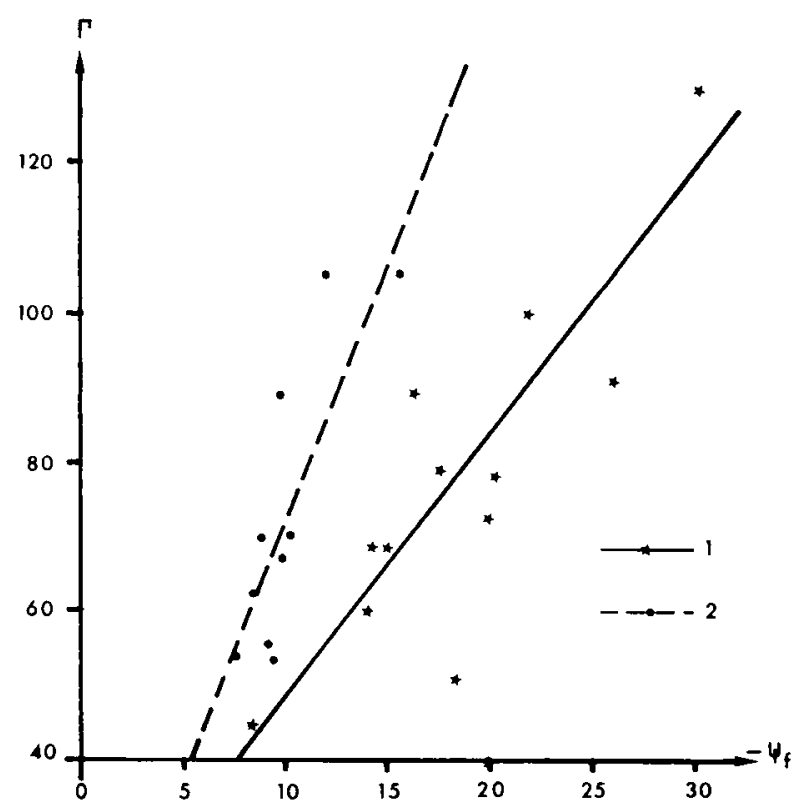

Figure 5

Evolution du point de compensation pour le $\mathrm{CO}_{2}(\Gamma$, vpm) en fonction du potentiel hydrique foliaire $(4 f$, bars) au stade $7-8$ feuilles.

(1) Bidi $17 \quad \Gamma=11,86+3,56(-\psi f) \quad r=0,84^{x x}$

(2) Capitole $\Gamma=5,16+6,65(-\psi / f) \quad r=0,90^{x x}$

Pentes des droites de régression significativement différentes au seuil de $1 \%$.

Relationship between $\mathrm{CO}_{2}$ compensation concentration $(\Gamma, v p m)$ and leaf water potential $(\psi f)$ at the 7-8-leaf stage. Slopes of the regression lines $\Gamma=\mathrm{A}+\mathrm{B}(-\psi f)$ of the two cultivars significantly different at $P<0.01$. 
variété sensible à la sécheresse dont la photosynthèse nette diminue fortement pour des potentiels hydriques bas.

\section{Rendement lumineux maximal}

Les mesures sont réalisées au stade végétatif (78 feuilles) et montrent, parallèlement à la chute de la photosynthèse nette au palier de saturation lumineux, une diminution $\mathrm{du}$ rendement lumineux maximal, déterminé par la pente à l'origine de la courbe $\mathrm{P}_{\mathrm{N}} / \mathrm{E}$. $\mathrm{La}$ valeur de l'éclairement saturant diminue avec le déficit hydrique, le point de compensation pour la lumière augmentant parallèlement.

"Capitole " présente une forte réduction du rendement lumineux maximal pour des potentiels hydriques foliaires compris entre - 10 et - 15 bars, "Bidi 17 » conservant des valeurs élevées au-delà de -20 bars (fig. 6).

\section{CONCLUSION ET DISCUSSION}

La réduction de la photosynthèse, et par conséquent celle des rendements au cours du déficit hydrique, est généralement attribuée, pour une large part, à la fermeture des stomates (BOYER, 1970 ; SHEARMAN et al., 1972 ; JOHNSON et al., 1974). Ces différents auteurs relèvent un parallélisme entre l'augmentation de la résistance foliaire et la chute de la photosynthèse. Les 2 dernières équipes montrent que l'activité de la PEP carboxylase et de la RUDP carboxylase, mesurée in vitro chez le sorgho et le blé, est peu affectée au cours d'un déficit hydrique.

Cependant, d'autres auteurs ont mis en évidence des effets du déficit hydrique sur les facteurs métaboliques : HANSEN (1971) note un accroissement de la résistance intracellulaire lié à une augmentation de la respiration et à un ralentissement des processus de carboxylation ; LAWLOR (1976) observe que l'enrichissement de la concentration en $\mathrm{CO}_{2}$ de l'air n'améliore pratiquement pas le taux de photosynthèse du blé et du tournesol, ce qui indique que le processus de limitation de la diffusion du $\mathrm{CO}_{2}$ n'est pas le seul à intervenir. Ce même auteur, en 1979, montre que l'activité de la RUDP carboxylase, peu affectée par un déficit hydrique moyen, diminue fortement pour des potentiels hydriques foliaires bas.

De même, l'activité de Hill et la photophosphorylation sont altérées au cours du déficit hydrique chez plusieurs espèces : cotonnier (FRY, 1970), tournesol (BOYER, 1971 ; KECK \& BOYER, 1974).

Nos résultats montrent que le déficit hydrique, chez le blé, affecte les phénomènes stomatiques et non stomatiques de la photosynthèse. Le rendement lumineux maximal diminue parallèlement à la conductance stomatique pour des gammes de potentiels hydriques foliaires nettement différentes chez les 2 variétés, en particulier au stade végétatif. Il semble cependant que chez la variété "Capitole", sensible à la sécheresse, l'importance des phénomènes non stomatiques soit plus grande et qu'ils apparaissent plus tôt par rapport à la fermeture stomatique (fig. 6). Ce fait permet de penser que les phénomènes non stomatiques peuvent

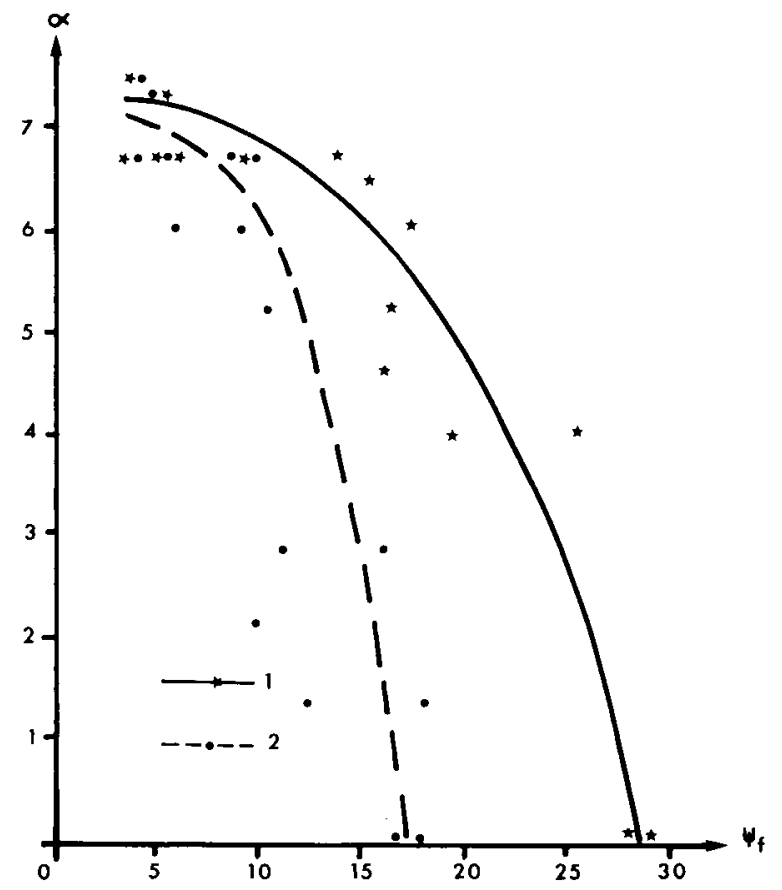

Figure 6

Relation entre le rendement lumineux maximal de la photosynthèse $\left(\alpha, 10^{-9} \mathrm{~kg} \mathrm{~J}^{-1}\right)$ et le potentiel hydrique foliaire $(\psi f$, bars $)$ au stade 7-8 feuilles.

$\begin{array}{ll}\text { (1) Bidi } 17 & \text { (2) Capitole. }\end{array}$

Relationship between maximum efficiency of light energv conversion $\left(x, 10^{-9} \mathrm{~kg} \mathrm{~J}^{-1}\right)$ and leaf water potential $(\psi f$, bar) at the 7-8-leaf stage.

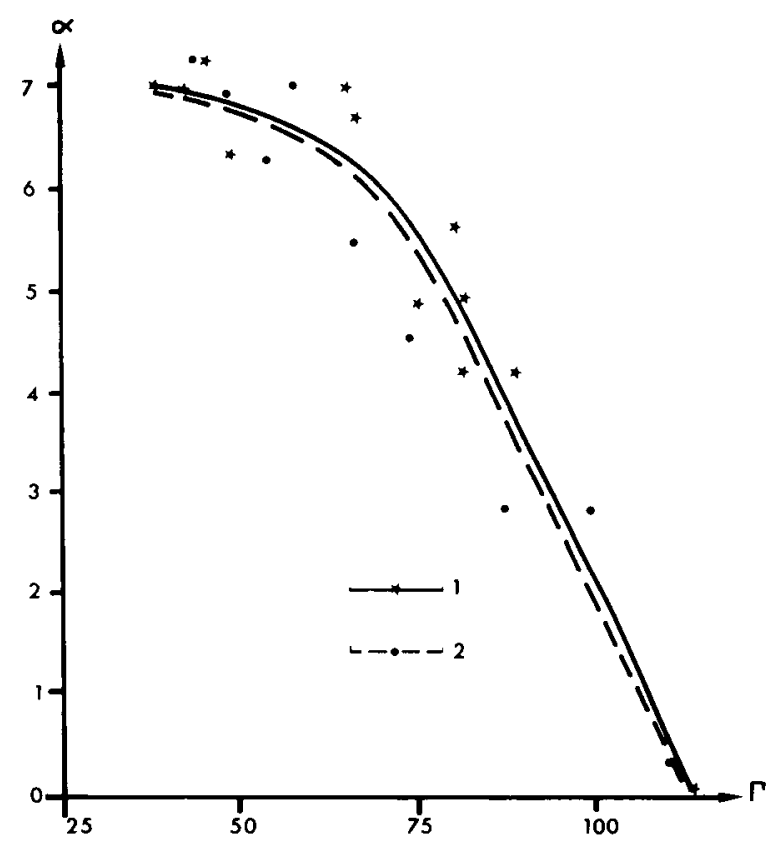

Figure 7

Relation entre le rendement lumineux maximal de la photosynthèse $\left(x, 10^{-9} \mathrm{~kg} \mathrm{~J}^{-1}\right)$ et le point de compensation pour le $\mathrm{CO}_{2}(\Gamma, v p m)$ au stade 7-8 feuilles. $\begin{array}{ll}\text { (l) Bidi } 17 & \text { (2) Capitole. }\end{array}$

Relationship between maximum efficiency of light energy conversion $\left(x, 10^{-9} \mathrm{~kg} \mathrm{~J}^{-1}\right)$ and $\mathrm{CO}_{2}$ compensation concentration $(\mathrm{T}$, vpm) at the 7-8 leaf stage. 
survenir relativement rapidement pour les variétés sensibles et pas uniquement dans ce cas pour les déficits hydriques sévères (LAWLOR, 1976).

Les phénomènes de carboxylation apparaissent nettement affectés ou du moins de façon plus importante que ceux de la décarboxylation liée à la photorespiration comme le montre l'évolution du point de compensation pour le $\mathrm{CO}_{2}(\Gamma)$, en fonction du potentiel hydrique foliaire.

La relation similaire (fig. 7) entre le rendement lumineux maximal $\alpha$ et le point de compensation pour le $\mathrm{CO}_{2}(\Gamma)$ obtenue pour les 2 variétés à des niveaux de potentiels hydriques foliaires nettement différents, démontre bien le poids de ces phénomènes de carboxylation et de décarboxylation dans l'expression du rendement lumineux maximal. Cependant, une altération des membranes chloroplastiques au cours du déficit hydrique peut intervenir dans la diminution du rendement lumineux maximal (HAVAUX \& LANNOYE, 1983), les processus photochimiques, la réaction de
Hill et la photophosphorylation étant parallèlement affectés (NIR \& POLJAKOFF-MAYBER, 1967; FRY, 1970 ; PHAM THI, 1976 ; BOYER, 1976).

Notre étude montre que le classement agronomique des 2 variétés pour la résistance à la sécheresse est confirmé par l'analyse des relations échanges gazeux foliaires - potentiel hydrique foliaire. L'ensemble des paramètres étudiés qui déterminent le niveau de la photosynthèse nette est favorable à la variété la plus résistante. Il est ainsi possible d'envisager la prise en compte d'un seul de ces facteurs dans le cadre d'une sélection des blés pour la résistance à la sécheresse. Compte-tenu des dispositifs de mesures rapides, la relation entre la résistance stomatique de face adaxiale de la feuille et le potentiel hydrique foliaire, pour un état hydrique du sol, peut être, de préférence, retenue.

Reçu le 24 avril 1984. Accepté le 15 mars 1985.

\section{RÉFÉRENCES BIBLIOGRAPHIQUES}

Begg J. E., Turner N. C., 1976. Crop water deficits. $A d v$. in Agron., 28, 161-217.

Boyer J. S., 1970. Differing sensitivity of photosynthesis to low leaf water potentials in corn and soybean. Plant Physiol., 46, 236-239.

Boyer J. S., 1971. Non-stomatal inhibition of photosynthesis in sunflower at leaf water potentials and high light intensities. Plant Physiol., 48, 532-536.

Boyer J. S. 1976. In T. T. Kozlowski : Water Deficits and Plant Growth. Vol. IV, 153-190, New York and London, Academic Press, $383 \mathrm{p}$.

Cornic G., Prioul J. L., Louason G., 1983. Stomatal and nonstomatal contribution in the decline in leaf net $\mathrm{CO}_{2}$ uptake during rapid water stress. Physiol. Plant., 58, 295-301.

Frank A. B., Power J. E., Willis W. O., 1973. Effect of temperature and plant water stress on photosynthesis, diffusion resistance and leaf water potential in spring wheat. Agron. J., 65, 777-780.

Fry K. E., 1970. Some factors affecting the Hill reaction activity in cotton chloroplasts. Plant Physiol., 45, 465-469.

Hansen G. K., 1971. Photosynthesis, transpiration and diffusion resistance in relation to water potential in leaves during water stress. Acta Agric. Scand., 21, 163-171.

Havaux M., Lannoye R., 1983. Chlorophyll fluorescence induction: a sensitive indicator of water stress in maize plants. Irrig. Sci., 4, 147-151.

Hsiao T. C., 1973. Plant responses to water stress. Annu. Rev. Plant Physiol., 24, 519-570.

Johnson R. R., Frey N. H., Moss D. N., 1974. Effect of water stress on photosynthesis and transpiration of flag leaves and spikes of barley and wheat. Crop Sci., 14 (5), 728-731.

Keck R. W., Boyer J. S., 1974. Chloroplast response to low leaf water potential. Plant Physiol., 53, 474-479.

Lawlor D. W., 1976. Water stress induced changes in photosynthesis, photorespiration, respiration and $\mathrm{CO}_{2}$ compensation concentration of wheat. Photosynthetica, 10 (3), 378-387.

Lawlor D. W., 1979. Effects of water and heat stress on carbon metabolism of plants with $\mathrm{C}_{3}$ and $\mathrm{C}_{4}$ photosynthesis. In $\mathrm{H}$. Mussel and R. C. Staples: Stress Physiology in Crop Plants, 303-326, Wiley Interscience, New York, $510 \mathrm{p}$.
Morgan J. M., 1977. Changes in diffusive conductance and water potential of wheat plants before and after anthesis. Aust. J. Plant Physiol., 4, 75-86.

Nir L., Poljakoff-Mayber A., 1967. Effect of water stress on the photochemical activity of chloroplasts. Nature, 213 (5074), 418-419.

Pham Thi A. T., 1976. Quelques aspects de l'influence de la carence hydrique sur la photosynthèse, la photorespiration et la respiration obscure chez deux espèces de cotonnier, l'une résistante à la sécheresse, l'autre sensible. Thèse Doct. d'Etat, Univ. Paris VII, 169 p.

Planchon C., 1976. Essai de détermination de critères physiologiques en vue de l'amélioration de blé tendre: les facteurs de la photosynthèse de la dernière feuille. Ann. Amélior. Plant., 26 (4), 714744.

Quarrie S. A., Jones H. G., 1979. Genotypic variation in leaf water potential, stomatal conductance and abscissic concentration in spring wheat subjected to artificial drought stress. Ann. Bot., 44 (3), 323-332.

Scholander P. F., Hammel H. T., Bradstreet E. D., Hemmingsen E. A., 1965. Sap pressures in vascular plants. Science, 148, 339-346.

Sharkey T. D., Badger M. R., 1982. Effects of water stress on photosynthetic electron transport, photophosphorylation and metabolic levels of Xanthium strumarium mesophyll cells. Planta, 156, 295301.

Shearman L. L., Eastin J. D., Sullivan C. T., Kinbacher E. J., 1972. Carbon dioxide exchange in water stressed sorghum. Crop Sci., 12, 406-408.

Simmelsgaard S. L., 1976. Adaptation to water stress in wheat. Physiol. Plant., 37, 167-174.

Turner N. C., Begg J. E., Rawson H. M., English S. D. et Hearm A. B., 1978. Agronomic and physiological responses of soybean and sorghum crops to water deficit. III. Components of leaf water potential, leaf conductance, $\mathrm{CO}_{2}$ (carbon dioxide isotope) photosynthesis and adaptation to water deficits. Aust. J. Plant Physiol., 5 (2), 179-194.

Watson B. T., Wardlaw I. F., 1981. Metabolism and export of ${ }^{14} \mathrm{C}$ labelled photosynthate from water-stressed leaves. Aust. J. Plant Physiol., 8, 143-153. 